\title{
An introduction of the Group AHP to the Personnel Assessment and its Direct and Indirect Influences on the Company's Performances ${ }^{1}$
}

\author{
Manabu Sugiyama \\ Department of Advanced Social and International Studies, University of Tokyo \\ 3-8-1 Komaba, Meguro-ku, Tokyo 153-8902, Japan \\ sugi@waka.c.u-tokyo.ac.jp
}

\begin{abstract}
In order to support group-consensus building, in the previous paper my colleagues and I have developed a new method of group AHP that is named "the Consensus Building Model by group AHP (CBM)." CBM is based on three important assumptions (hypotheses) for human behavior towards the consensus of a group. We developed a personnel assessment support tool of system engineers using CBM and made a personnel assessment for a system integration company.

The purpose of this paper is to show how to raise company's productivity by introducing the CBM-based personnel assessment. An introduction of the group AHP to personnel assessment can improve company's productivity directly or indirectly. I suggest that the consensus of assessors is a key factor influencing the productivity. Here the consensus enhances the shared recognition of employees about of the cooperate infrastructure, clarifies the process for solving the internal decision problems of a company, and thus optimize the cooperate infrastructure. This result suggests that the effective use of OR methods is not limited to a specific problem-solving, but it extends to a self-evaluation of the company's infrastructure, such as personnel decisions.
\end{abstract}

\section{Introduction}

In the last few decades, the corporate employment systems in Japan have changed rapidly and radically. We expect that a new concept of employment will be established by the beginning of the 21st century. In the past, most Japanese employment systems were well known to be seniority-based. In contrast, in the USA and Europe, merit-based employment is most common. These changes in Japan clearly indicate a transition from the Japanese traditional seniority-based systems to the newer merit-based systems. However, in Japan, most important cooperate decisions such as personnel decisions are made under consensus of the top people unlike the USA and Europe, where decisions are mostly made by a single (top) person in a company. Therefore, in Japan, a fair assessment of employees' performance focusing on strong points will become a major issue for management in the near future. This is particularly important in the software industry, where drastic changes are currently on-going dramatically (Labor \& Employment Committee, 1995).

In order to support group-consensus building, a new method of the Consensus Building Model had been developed using group AHP (CBM) (Yamada, Sugiyama and Yamaki, 1997). Then using $\mathrm{CMB}$, a new personnel assessment support tool for system engineers has been developed and used as a practical tool for personnel assessment in a system integration company. Here I call the company "SIC." SIC is a company that has been growing rapidly in software industry. Currently it has about 100 systems engineers, and its annual sales are approximately US\$10 million. SIC has been approved as a system integrator by the Ministry of International Trade and Industry (MITI) of Japan.

${ }^{1}$ Acknowledgment: I would like to thank Mr. Shuntarou Shimada, President of the Research Institute of Systems Planning (ISP) for supporting our research, Mr. Tokimune Koh, systems engineer of the ISP, for developing the software. In addition to above, I would like to thank Professor Yoshiyasu Yamada and Professor Naokazu Yamaki for discussion and useful suggestion. 
SIC's systems engineers are required to have both highly technical knowledge and the ability to develop software. SIC recognizes and promotes personal achievements under the motto: "Creativity, Advancement and Engineer's Spirit." Most of SIC's systems engineers have a pride and confidence as an able engineer. Therefore, it is extremely important to use a sound personnel assessment system that is valid and accepted by these systems engineers. In a recent speech, SIC's president said that a good personnel assessment that is credible to systems engineers can enhance their morale, improve productivity and as a result promote the development of the company.

\section{The previous personnel assessment and its problems}

Following many years of discussion, the board of directors of SIC arrived at a consensus that the personnel assessment credible to systems engineers should meet certain conditions. First, the evaluation process should be conducted scientifically and transparently, rather than trying to make the assessment results fair. This is because the personnel assessment results inevitably vary or are influenced by the values of the person making the assessment. The board acknowledged that most SIC's systems engineers, being engineers, know the merits and demerits of the scientific process. Thus, though they may not necessarily be convinced of the evaluation result, they would nevertheless accept the evaluation process so long as it is scientific.

Second, in order to make the systems engineer's personnel assessment as fair as possible, the board set three steps for the assessment process. First, the scientific evaluation system is used to assess the systems engineer, and the result thus obtained is taken as the preliminary evaluation. Then, the group of evaluators discusses this preliminary evaluation so that the primary assessment result of each systems engineer may be modified to minimize any bias of the individual evaluator. Finally, further adjustments may be made by the board of directors, taking company policy into consideration, and the final assessment result is worked out.

Based on this agreement by the board of directors, SIC's previous personnel assessment system included the following procedure.

[Step 1] For the preliminary evaluation, the evaluator (usually the project manager to whom the systems engineer directly reports) evaluates the systems engineer by use of the "decision hierarchy" as shown in Figure 1. Weighting among evaluation items at each level of the decision hierarchy is decided each year by the board of directors. The preliminary evaluation of each systems engineer is decided at this step.

[Step 2] In the group of evaluators, the evaluation score, calculated for each systems engineers by the project manager/evaluator, is compared with those of other evaluators calculated for the systems engineer in question. Thus differences between scores of various evaluators are adjusted. Then, the obtained result is fixed as the preliminary assessment result for the systems engineer in question.

[Step 3] The board of directors then adjusts (if necessary) each systems engineer's preliminary assessment results to arrive at the final assessment result. Since this assessment result directly affects salary increases and bonuses, it has been a subject of great concern among systems engineers.

The personnel assessment system for systems engineers has incorporated the procedure described above. This system has been accepted by almost all SIC's systems engineers. However, the board of directors, project managers and systems engineers have asked for improvements based on the following points.

i) The weighting of evaluation items was decided by the board of directors. However, the weighting varied considerably from one director to another. Out of necessity, the board nevertheless decided on one single weighting. The board wanted to clarity the consensus building process to decide on one single weighting among directors.

ii) The system should be improved so that evaluations are easier to make. To see which systems engineer of the pair is better at personnel evaluation, paired comparison has been adopted by SIC as a system to make a relatively accurate evaluation. Board members felt it would 


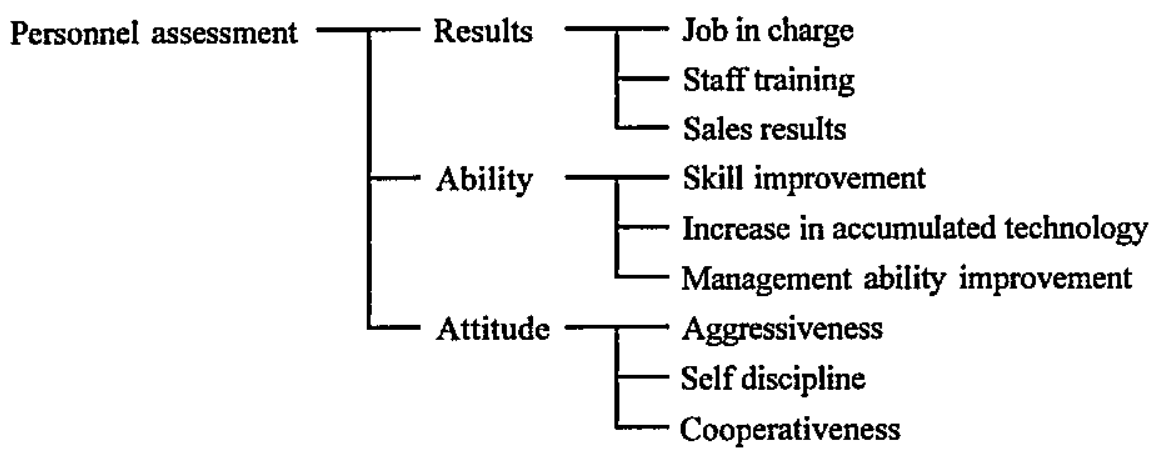

Figure 1: Decision hierarchy for evaluating systems engineer

be easier to make a more accurate evaluation if the paired comparison were incorporated into the evaluation system. (Speech by president)

iii) The board of directors, has repeatedly discussed the possibility of working out a system to clarify the group evaluation process.

iv) The company has been hoping to establish a personnel assessment system that maximizes group satisfaction, said to be one of the advantages of the Japanese corporate management approach. Moreover, Nonaka has pointed out that the advantage of this Japanese approach is "middle-up-down management." Thus the most desirable personnel assessment system would enable maximum participation by middle management (Nonaka, 1988).

SIC has been studying a system of personnel assessment to solve the problems mentioned above. At that time, our research group developed CBM as one of the group AHP methods (Yamada, Sugiyama and Yamaki, 1997).

Based on the thinking that CBM could be useful as a personnel evaluation method at SIC, CBM was introduced as an experiment in 1995. Today, CBM still serves as a useful tool at SIC. A more detailed description follows.

\section{The Consensus Building Model by group AHP}

The Analytic Hierarchy Process (AHP) shown to be an effective method for subjectively weighting evaluation items. In addition, a method to apply AHP to group decision making has been proposed by Saaty (Saaty, 1989). Our research group proposed the use of a group consensus-building method incorporating such factors as strength of individual opinion and compromise (Yamada, Sugiyama and Yamaki, 1997). We call this method CBM.

CBM is a method for group consensus-building based on the following three hypotheses about humans.

[Hypothesis 1] Humans are more willing to revise their judgment before this judgment is firmly established. On the other hand humans resist being forced to change a judgment after it has been firmly established.

[Hypothesis 2] It is easier for humans to accept and agree to a judgment when it does not conflict with other judgments.

[Hypothesis 3] It is easier for each member of the group to agree with the group decision if there are fewer differences between the group decision and his or her own decision.

The above hypotheses about humans may be typical of and unique to the Japanese culture. In any case, they have been empirically accepted in Japan where group consensus forms the foundation of decision making (Matsuda, 1978).

In an evaluation process with CBM, each member of a group determines his or her own pairedcomparison element as a first step. In this step, when the group obtain each member's evaluation 
by questionnaire or others, it may be suggested that each member should be allowed to have some latitude in his thinking so as to facilitate the building of a group consensus. That is to say, each member is instructed to describe the paired-comparison element by "interval," rather than "point." Therefore, we call this an interval for assertion (IA), which is an interval of pairedcomparison element established by individual members. In other wards, CBM includes IA in order to use hypothesis 1 .

The IA represents each member's evaluation value and the strength of assertion. We denote $k$-th member's IA from the item " $j$ " to the item " $i$ " by $I A_{i j}^{(k)}$. Where the number of group members is " $m$," and $I A_{i j}^{(k)}=\left[l_{i j}^{(k)}, u_{i j}^{(k)}\right]$, the paired-comparison matrix of the group is determined as $\boldsymbol{X}=\left(x_{i j}\right), x_{i j}=\left[\tilde{l}_{i j}, \tilde{u}_{i j}\right]$. In addition to above, the details for determination of the group paired-comparison matrix $\boldsymbol{X}$ are described in the paper of CBM (Yamada, Sugiyama and Yamaki, 1997).

The CI-DI model to determine the weight vector $\boldsymbol{w}=\left(w_{1}, \ldots, w_{n}\right)^{\mathrm{T}}$ of evaluation items, is indicated as follows:

$$
\begin{aligned}
& \min \alpha(C I)+\beta(D I), \\
& \text { subject to } \sum_{j=1}^{n} x_{i j} w_{j}=\lambda w_{i} \quad(i=1, \ldots, n) \text {, } \\
& x_{i j} x_{j i}=1 \quad(i, j=1, \ldots, n), \\
& \sum_{i=1}^{n} w_{i}=1 \\
& w_{i}>0 \quad(i=1, \ldots, n), \\
& \tilde{l}_{i j} \leq x_{i j} \leq \tilde{u}_{i j} \quad(i, j=1, \ldots, n) .
\end{aligned}
$$

Where, $\alpha$ and $\beta$ are parameter as priority, and $\lambda$ is the maximum eigenvalue of the group pairedcomparison matrix $\boldsymbol{X} . C I$ is the consistency index (C.I.), and CBM includes $C I$ in order to use hypothesis 2 .

$$
C I=\frac{\lambda-n}{n-1}
$$

$D I$ is the dissatisfaction index as a degree of satisfaction, and CBM includes $D I$ in order to use hypothesis 3 .

$$
\left.\begin{array}{rl}
D I= & \frac{D S-M D S}{M D S}, \\
D S=\sum_{i<j} \sum_{k=1}^{m} d_{i j}^{(k)}\left(\ln x_{i j}-\ln c_{i j}^{(k)}\right)^{2}, \\
M D=\sum_{i<j} \sum_{k=1}^{m} d_{i j}^{(k)}\left(\ln p_{i j}-\ln c_{i j}^{(k)}\right)^{2}, \\
\ln p_{i j}=\frac{1}{\sum d_{i j}^{(k)}} \sum_{k=1}^{m} d_{i j}^{(k)} \ln c_{i j}^{(k)}, \\
d_{i j}^{(k)}=\left|\ln u_{i j}^{(k)}-\ln l_{i j}^{(k)}\right|, \\
c_{i j}^{(k)}=\sqrt{l_{i j}^{(k)} \cdot u_{i j}^{(k)}} .
\end{array}\right) .
$$

CBM is one AHP method. Therefore, as has been said for traditional AHP, CBM is useful for clarifying the process of decision making (Takai, 1990). It also facilitates more accurate evaluation of a number of items by using paired comparison evaluation. When multiple evaluators use AHP in making their evaluations, it clarifies the evaluation process of other evaluators. This benefit alone makes AHP useful for group consensus-building. The AHP process of CBM likewise clarifies the process of group consensus-building. Since many middle managers participate in the evaluation decision, through CBM the decision reflects their values more accurately. In this way, the company gains maximum from Japanese-style management. 
For these reasons, CBM was introduced experimentally with the expectation that it would be effective in solving the problems of SIC personnel assessments.

\section{Implementing CBM to support SIC's personnel assessment}

CBM was used experimentally to evaluate systems engineers for bonuses in the fall of 1995 as well as the spring and fall of 1996. Prior to the experiments, Professor Yamada and I met several times with systems engineers - both evaluators and those to be evaluated. They received lectures and explanations about the concept of AHP and CBM, the theoretical basis, characteristics of the method, success stories at other companies, etc. Since systems engineers at SIC excel in highly technical knowledge and mathematical ability, it seemed that they had fully understood AHP and CBM after these several lectures. However, during the first experiment, it became clear that not all the evaluators fully understood AHP. This became evident when data which did not accurately reflect the opinion of the tested persons were included due to a lack of understanding of AHP.

Table 1: CBM implementation process

\begin{tabular}{ccc}
\hline & The time of implementation & Contents of implementation \\
\hline The first experiment & Fall, 1995 & Understanding of CBM \\
The second experiment & Spring, 1996 & Improvement of CBM \\
The third experiment & Fall, 1996 & Expansion of CBM \\
\hline
\end{tabular}

This first experiment was carried out in the fall of 1995, immediately after about 20 middle managers evaluated systems engineers regarding bonuses. The same evaluators then evaluated systems engineers using CBM as well as the same decision hierarchy as before. An analysis showed that most evaluation results of systems engineers by each evaluator using CBM were very similar to those evaluated by the traditional method. However, several results showed large differences between these two methods. Then, we analyzed why such large difference occurred between the CBM evaluation and those of the traditional evaluation.

The analysis showed that some assessment results increased the preliminary evaluation scores, because intangible factors not included in the decision hierarchy were evaluated highly. We reported the results of this analysis to the president and board of directors of the company and gave them a clearer understanding of the system. As a result, the company agreed that we would carry out a similar experiment for the bonus evaluations in the spring of 1996.

For this second experiment, we created AHP questionnaire software based on our experience in the first experiment, and we proposed a simplified evaluation method. One interesting fact was discovered during the experiment. Since there was excessive difference in the weighting of evaluation items by evaluators, we charted the results for evaluators and asked them to modify the weighting. However, most of them did not change their own weighting. From this, we concluded that weighting expresses the values and strong convictions of each evaluator. Consequently, we thought that the consensus among evaluators resulted from the rationality of the evaluation process, rather than from the fairness of the evaluation result.

In the fall of 1996, the third experiment was carried out for bonus evaluations in order to make the systems engineers assessment system more practical. Based on the results of the previous two tests, the AHP questionnaire software was further improved, and CBM was also improved so as to calculate more realistic results. As a result, CBM was accepted as a very useful supporting tool for systems engineers personnel assessment. More precisely, as to the use of CBM as a personnel assessment tool appraised varied depending on the person's position in the company. However, almost everybody agreed that CBM is effective as a supporting tool for personnel assessment.

After these three experiment, SIC now is successfully using CBM as a personnel assessment supporting tool. A problem currently encountered by using is that CBM requires too much work to evaluate many systems engineers by AHP. Improving this aspect will make CBM an even more practical and useful tool. 


\section{Conclusions}

After carrying out the experiments using CBM in the process of systems engineer personnel assessment at SIC, we made an analysis of the CBM results as useful information for the assessment. We learned the following.

\section{[Direct influence]}

i) CBM was accepted as a very useful supporting tool for systems engineers personnel assessment.

[Evidence] The results obtained by using the OR method such as CBM should be used as information necessary for decision making rather than being directly used for decision making or the evaluation decision. That is because in the decision making results from the OR method, influence of intangible factor which is difficult to be taken up as evaluation item tends to be neglected. Actual decision making should include not only the calculated results from the OR method, but also the experience and intuition of the decision maker.

ii) It has become clear that CBM is useful in solving the company's problems when utilized within the process of personnel assessment that consists of preliminary personnel evaluation, primary assessment by evaluator group and final assessment by the board of directors.

[Evidence] We have learned that the OR method can be continually improved after understanding the difference between the actual problem and model problem in the OR application cycle.

\section{[Indirect influence]}

i) We have seen that the OR process, like CBM, is useful to deepen common recognition in the group to clarify the evaluation structure for personnel assessment.

[Evidence] This is clear from the fact that the adoption of CBM activated discussions among evaluators as to evaluation decision hierarchy and weighting of evaluation items, and clarified the structural process of personnel assessment evaluations.

ii) From these experiments we found out that assuring the fairness of the evaluation process is vital to having systems engineers accept the results.

[Evidence] We also learned that introducing the OR process enabled systems engineers to understand that the company tries to fairly carry out the evaluation process, rather than providing its own solution.

From the above, it is possible to say that the OR process is an effective method for structuring problems, clarifying the process and enhancing the common recognition of the problems when it is incorporated in the business system, rather than being used for the solution of the company's problems.

\section{References}

Labor \& Employment Committee (1995), Proposal of New Employment System for Information Service Industry, Japan Information Service Industry Association (in Japanese).

Matsuda,T. (1978), "System Model of OR Implementation and Japanese Organization Climate" (in Japanese), Communications of the Operations Research Society of Japan 23, 668-672.

Nonaka,I. (1988), "Toward Middle-Up-Down Management : Accelerating Information Creation," Sloan Management Review 29.

Saaty,T.L. (1989), "Group Decision Making and The AHP," in: B.L.Golden, E.A.Wasil and P.T.Harker (eds.), The Analytic Hierarchy Process, Springer-Verlag, 59-67.

Takai,E. (1990), "Consensus Building of Mainframe Computer Selection of the Company" (in Japanese), in: Tone,K. and Manabe,R. (eds.), Collection of AHP Application (in Japanese), JUSE Press, Ltd., 204-218.

Yamada,Y., Sugiyama,M. and Yamaki,N. (1997), "Group Analytic Hierarchy Process Based on Consensus Making Model" (in Japanese), Journal of the Operations Research Society of Japan 40, 236-244. 